Тучина Дарья Сергеевна, Старченко Алексей Валерьевич, Стреб̆кова Татьяна Владимировна, Питько Юлия Михайловна

\title{
ПРОВЕРКА ФУНКЦИОНАЛЬНОСТИ СЕТИ НА ПРЕДМЕТ ОБМЕНА ДАННЫМИ МЕЖДУ УСТРОЙСТВАМИ НА ЦИФРОВОЙ ПОДСТАНЦИИ
}

Во всем мире большое внимание уделяется новой коммуникационной технологии МЭК 61850. Особое место занимает программа иифровизации электросетевого комплекса РФ, согласно которой уже к 2025 году будут функционировать около 40 циифровых подстанций. Обслужсивание таких систем по сравнению с традиционными в целом проце, но к ним предъявляют несколько иные требования - требования к информационной сети. $K$ сожалению, стандарт описывает только требования, но не описывает алгоритиа проведения проверки.

В данной статье предложен механизм проверки функциональности архитектуры связи устройств на цифровой подстанции с помощьто аппаратно-программного комплекса, разработанного в среде LabVIEW, который позволяет проводить точную оценку соответствия сети предъявляемым требованиям.

Ключевые слова: МЭК 61850, цицрровая подстанция, GOOSE, MMS, SV.

\section{Daria Tuchina, Aleksey Starchenko, Tatyana Strebkova, Julia Pitko CHECKING THE FUNCTIONALITY OF A NETWORK ON DATA EXCHANGE BETWEEN THE DEVICES ON THE DIGITAL SUBSTATION}

Around the world, much attention is paid to the new communication technology IEC 61850. A special place is occupied by the program of Digitization of the electric grid complex of the Russian Federation, according to which 40 digital substations will function by the year 2025. Maintenance of such systems as compared with traditional ones is generally simpler, but they have somewhat different requirements requirements for the information network. Unfortunately, the standard describes only the requirements, but does not describe the verification algorithm.

This article proposes a mechanism for checking the functionality of the device communication architecture at a digital substation using an application of a hardware-software complex developed in the LabVIEW environment that allows an accurate assessment of network compliance with the requirements.

Key words: IEC 61850, digital substation, GOOSE, MMS, SV.

Bведение / Introduction. За последние годы в области электроэнергетики был сделан большой шаг вперед - во всем мире началось массовое внедрение цифровых подстанций (ЦПС), и эта тенденция набирает силу. Эффективность управления подстанцией нового поколения напрямую зависит от скорости передачи информационных потоков между ее объектами, поэтому данный вопрос имеет ключевое значение. Технологии «Цифровая подстанция» ведет к значительному повышению энергоэффективности и сокращению продолжительности простоев в случае возникновения аварийных ситуаций, связанных с работой многофункциональных защитных интеллектуальных электронных устройств.

В первой части описываются функции, состав цифровой подстанции и требования, предъявляемые к ним. Во второй половине исследования приведен механизм для тестирования оборудования подстанции на основе МЭК 61850 и то, как они отвечают требованиям, предъявляемым к скорости передачи данных и временным задержкам на цифровой подстанции.

Материалы и методы / Materials and methods. Цифровая подстанция (ЦПС) - это элемент активно-адаптивной электросети с системой контроля, защиты и управления, основанной на передаче информации в цифровой форме [1]. 
В зависимости от организации связи ЦПС подразделяются:

- с распределенной многотерминальной архитектурой - стандартная (рис. 1a);

- с централизованной архитектурой связи (рис. 1б);

- с кластерной архитектурой (рис. 1в).

В соответствии со стандартом МЭК 61850 система автоматизации энергетического объекта (ЦПС) делится на 3 уровня:

1) уровень станции - пункт управления подстанцией. Здесь осуществляется онлайн-мониторинг и анализ собранных данных. Состоит из серверов верхнего уровня (система SCADA, система телемеханики, сервер хранения данных), автоматизированных рабочих мест персонала подстанции (АРМ);

2) уровень ячейки - состав данного уровня зависит от исполнения архитектуры ЦПС. В стандартном варианте организации связи уровень ячейки включает в себя интеллектуальные электронные устройства (многофункциональные измерительные приборы, АСКУЭ, устройства релейной защиты и автоматики). Подобная схема организации изображена на рис. 1а. При централизованном исполнении архитектуры связи на уровне ячейки происходит разделение программного обеспечения от аппаратной части с реализацией функций защит и управления на общепромышленном сервере (рис. 1б). На рис. 1в изображена структура ЦПС в кластерном исполнении - уровень ячейки включает в себя универсальные терминалы с возможностью резервирования и распределения функций между устройствами в режиме реального времени (рис. 1в);

3) уровень процесса содержит первичное оборудование, цифровые / традиционные измерительные трансформаторы, MU - устройства сопряжения с шиной, УСО - устройства сопряжение с объектом (перевод дискретной информации в цифровой сигнал), SAMU устройства сопряжение с объектом (перевод аналоговой информации в цифровой сигнал), датчики для сбора аналоговой / дискретной информации [2], [3].

Главное условие эффективности ЦПС - использование международных стандартов, позволяющих обеспечить построение энергетических объектов на базе оборудования различных производителей [4].

Для обеспечения правильной работы ЦПС и ее компонентов необходимо провести испытания на соответствие техническим минимальным требованиям [5].

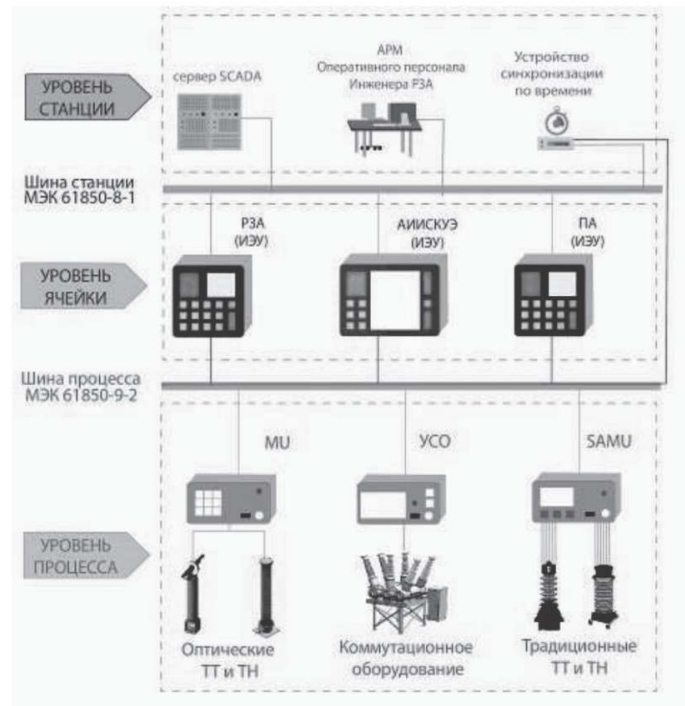

a)

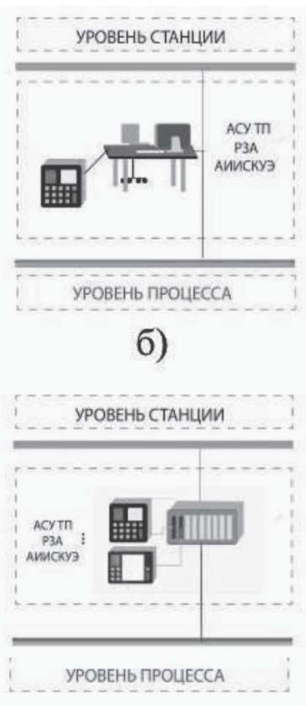

B)

Рис. 1. Разновидности архитектур связи на цифровой подстанции 
Стандарты, применяемые на цифровых подстанциях

\begin{tabular}{|c|c|c|}
\hline $\begin{array}{l}\text { Применение } \\
\text { стандарта } \\
\text { в условиях ЦПС }\end{array}$ & $\begin{array}{l}\text { Наименование } \\
\text { стандарта }\end{array}$ & Функции стандарта \\
\hline Шина процесса & МЭК-61850-9-2 LE & $\begin{array}{l}\text { Передача информации о мгновенных значениях тока и } \\
\text { напряжения от измерительных трансформаторов тока } \\
\text { и напряжения в формате потока данных SV }\end{array}$ \\
\hline $\begin{array}{l}\text { Шина станции, обмен } \\
\text { УСО }\end{array}$ & МЭК-61850-8-1 & $\begin{array}{l}\text { Передача сигналов управления коммутационными ап- } \\
\text { паратами в формате GOOSE-сообщений, а также орга- } \\
\text { низация информационного взаимодействия устройств } \\
\text { P3A c ACУ TП подстанции посредством протокола } \\
\text { MMS (данные телесигнализации и телеизмерений, пе- } \\
\text { редача команд телеуправления) }\end{array}$ \\
\hline Измерения & МЭК 61869 & Требования к трансформаторам тока и напряжения \\
\hline $\begin{array}{l}\text { Синхронизация } \\
\text { по времени }\end{array}$ & $\begin{array}{l}\text { МЭК } 61588 \\
\quad 1 \text { PPS }\end{array}$ & $\begin{array}{l}\text { Протокол точной тактовой синхронизации для сете- } \\
\text { вых систем измерения и управления }\end{array}$ \\
\hline $\begin{array}{l}\text { Проектирование и } \\
\text { инжиниринг }\end{array}$ & МЭК 61850-6 & $\begin{array}{l}\text { Требования к формату файлов описания конфигура- } \\
\text { ции специальных специфичных для систем связи ин- } \\
\text { теллектуальных электронных устройств }\end{array}$ \\
\hline $\begin{array}{l}\text { Информационная } \\
\text { безопасность }\end{array}$ & $\begin{array}{c}\text { МЭК 62351 } \\
\text { МЭК 61850-90-5 }\end{array}$ & $\begin{array}{l}\text { О требованиях к кибербезопасности ЦПС, примене- } \\
\text { ние аутентификации и шифрования }\end{array}$ \\
\hline
\end{tabular}

Проверка цифровой подстанции включает в себя:

1. Функциональность:

- проверка на соответствие оборудования стандарту МЭК 61850, возможность работы тестируемого оборудования с оборудованием цифровой подстанции;

- проверка коммуникационного стека на применимой шине (отклик, прохождение сигнала).

2. Надежность:

- проверка по критерию «N-1》 означает, что полная функциональность поддерживается при сбое какого-либо одного компонента. Сетевое подключение поддерживается, несмотря на любой отказ;

- время восстановления - один из критериев надежности, максимальное время возможного прерывания после сбоя без последствий для установки. На шине станции время восстановления после сбоя должно быть таким, чтобы время передачи GOOSE-сообщений не превысило критического порога;

- техническое обслуживание является необходимым условием для обеспечения надежности. На подстанции должна применяться комплексная стратегия обнаружения и обслуживания отказов, которая позволит минимизировать время ремонта.

3. Безопасность - рассматривая с позиции безопасности элементы цифровых подстанций следует отметить, что здесь любая подсистема содержит типовые интеллектуальные микропроцессорные программируемые компоненты, и чем больше мы получаем данных с приборов, чем проще становится процесс управления, тем острее становится вопрос безопасности. Специально для цифровых подстанций был разработан стандарт МЭК 62351, который предусматривает для защиты от несанкционированного доступа использование: аутентификации - криптографической подписи (при запросе доступа к системе диспетчер проходит аутентификацию с помощью криптоключа) и шифрования (применяется для передачи закрытой информации, например, показаний счетчика электроэнергии в систему АСКУЭ) $[6,7]$. 
4. Емкость канала связи - для обеспечения нормальной работы подстанции необходимо оценить пропускную способность канала. Важным шагом является измерение критических факторов, влияющих на производительность, таких как временная задержка.

Проверка технологического оборудования на предмет функциональности и соответствие техническим требования по быстродействию обмена информации производилась с помощью программного комплекса LabVIEW производства National Instruments - специальный пакет, поддерживающий МЭК 61850, на стандартном ПК - виртуальная часть установки, ИЭУ (микропроцессорный терминал) - вещественная часть установки, передача данных по различным протоколам передается по сети Ethernet с помощью сетевого коммутатора и встроенного в программу анализатора времени, позволяющего выявлять время задержки передачи данных между отправителем и получателем (рис. 2).

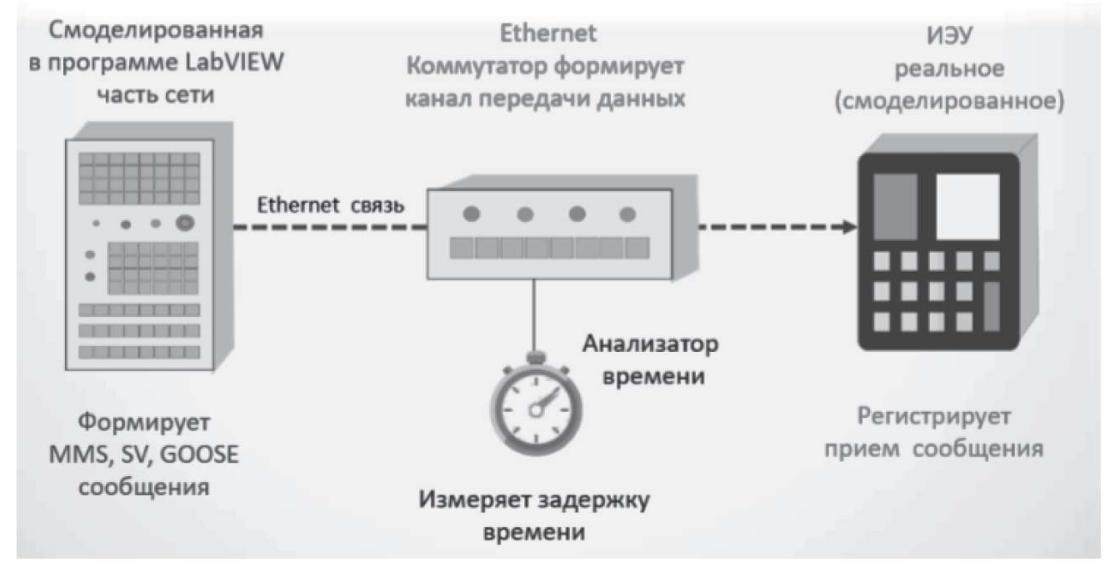

Рис.2. Установка для проведения эксперимента

Согласно международному стандарту организации ПАО «ФСК ЕЭС» № 96 (Типовые методики испытаний компонентов ЦПС на соответствие стандарту МЭК 61850 первой и второй редакции) и стандарту МЭК 61850 , для обмена данными между множеством устройств цифровых подстанций на различных уровнях взаимодействия, использование предлагаемых программных и аппаратных инструментов для тестирования элементов и каналов связи для систем, поддерживающих протокол МЭК 61850, является разрешенным [8].

Обмен информацией между тестовым и тестируемым устройством производится в цифровом виде по Ethernet-каналам посредством логических сигналов в виде посылки GOOSE сообщений (рис. 3).

Генерация и отсылка сообщений SV выполняется с заданием периода синхронизации временных меток, также возможно задание способа представления передаваемых данных в виде целочисленных значений либо численных значений с плавающей точкой. Очевидно, объем данных при представлении одного и того же сигнала будет разным, что дает возможность оценить возможности по загрузке канала передачи данных и принимающего данные оборудования на устойчивость к потере данных в условиях большой загруженности обрабатывающих приложений экспериментально худшие условия, которые могут повлиять на эффективность передачи данных в данной установке (рис. 4).

Таюже одним из инструментов тестирования является комплекс аппаратно-программного обеспечения, воспроизводящий технологию передачи сообщений MMS. Один из его возможных вариантов представления приведен на рис. 5. Передача обработанной информации от устройств шины процесса принимает компактный вид и несет в себе, как правило, сконцентрированную информацию о текущих параметрах режима, состоянии коммутационного оборудования и др. 


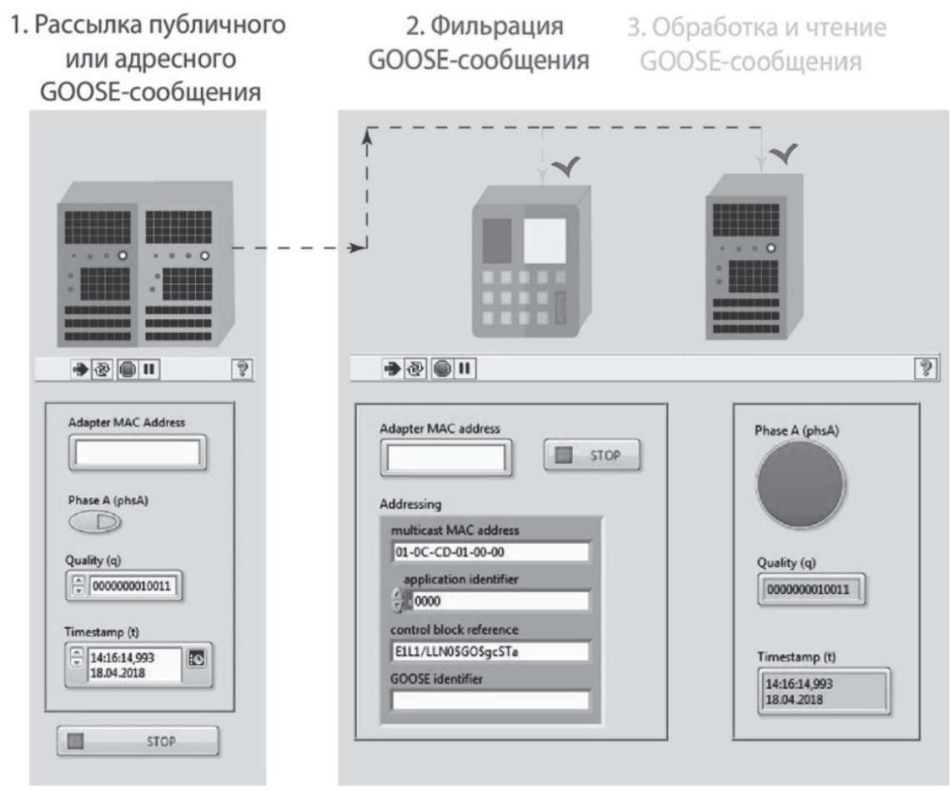

Рис. 3. Схема взаимодействий тестового и тестируемого устройств при посылке GOOSE-сообщений по стандарту МЭК 61850

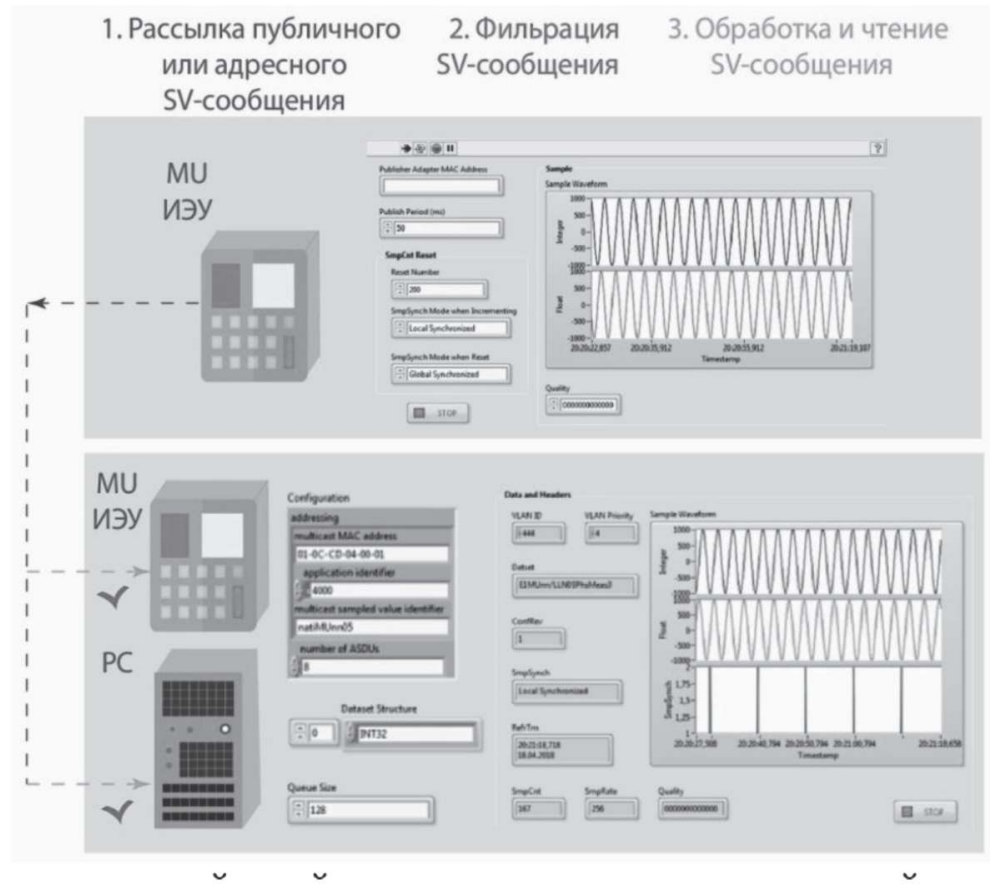

Рис. 4. Схема взаимодействий тестового и тестируемого устройств при посылке SV-сообщений по стандарту МЭК 61850

На приведенном рис. 5 показан процесс передачи значений векторов тока и напряжения трехфазной системы передачи электроэнергии. Измерение данных векторов производится интеллектуальным электронным устройством с жесткой привязкой измеренных значений токов и напряжений ко времени с последующим преобразованием синусоидального сигнала в векторное представление с организацией «посылки» в формате сообщений MMS и его передачей. 


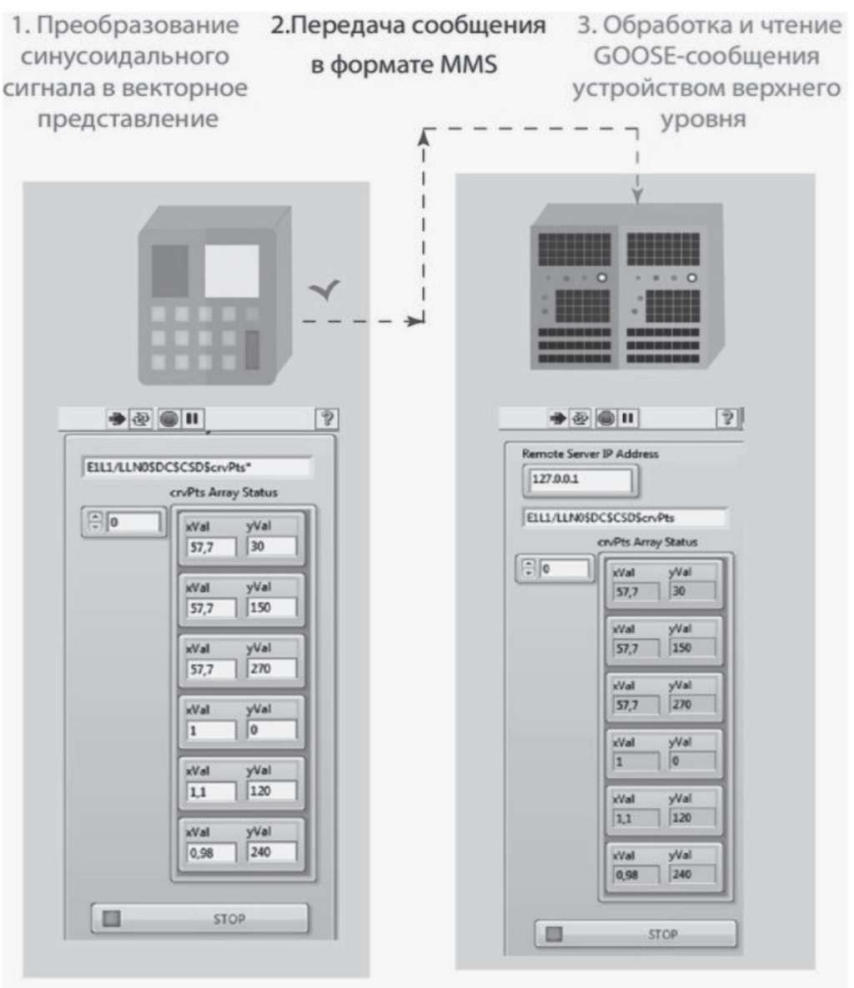

Рис. 5. Схема взаимодействий тестового и тестируемого устройств при посылке MMS-сообщений по стандарту МЭК 61850

Результаты и обсуждение / Results and discussion. На рис. 6 представлены результаты измерений временной задержки сигналов MMS для сети с различной скоростью сети Ethernet 100 мбит и 1 Гбит при различной загруженности информационной сети. На графике видно, что зависимости между временем задержки при передаче с использованием роутера со скоростью 100 Мбит и 1 Гбит не прослеживается (зависит от роутера). MMS использует двухстороннюю передачу, и максимальная временная задержка составляет 2,02 мсек при измерении времени в обе стороны и удовлетворяет всем критериям по производительности - согласно МЭК 61850, временная задержка должна быть не выше 3 мсек.

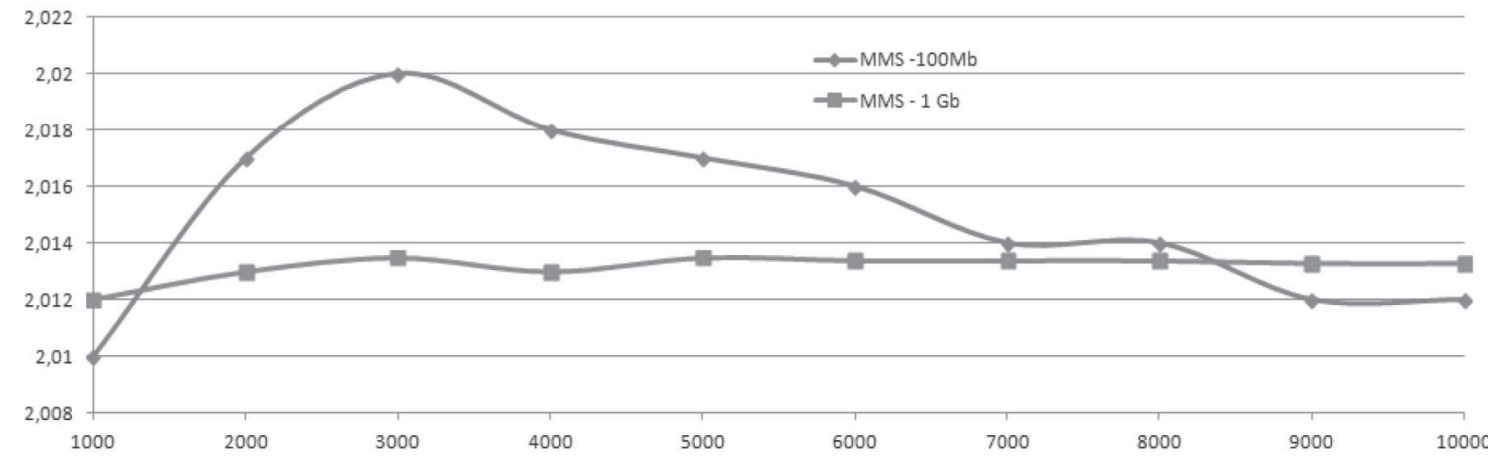

Рис. 6. Результаты измерений временной задержки сигналов MMS для сети

с различной скоростью сети Ethernet - 100 Мбит

и 1 Гбит - при различной загруженности информационной сети 
Заключение / Conclusion. В связи с массовым введением в эксплуатацию цифровых подстанций остро встает вопрос о проведении испытаний оборудования на соответствие техническим требованиям, предъявляемым задачами релейной защиты, систем АСДУ и др. устройств.

В данной статье предложен механизм проверки с помощью аппаратно-программного комплекса, разработанного в среде LabVIEW, позволяющего проводить точную оценку соответствия архитектуры связи ЦПС предъявляемым требованиям. Одним из них является оценка временной задержки - согласно проведенному эксперименту использование данного метода допустимо. В дальнейшем планируется усложнение эксперимента путем добавления дополнительных элементов - для увеличения загрузки сети, но прежде необходимо расширение пропускного канала с помощью более мощного роутера.

\section{ЛИТЕРАТУРА И ИНТЕРНЕТ-РЕСУРСЫ}

1. Аношин А. О., Головин А. В. Стандарт МЭК 61850. Протокол GOOSE // Новости ЭлектроТехники. 2012. № 6(78). Протокол МЭК 61850 Коммуникационные сети и системы подстанций.

2. МЭК 61850-8-1(2011) Сети связи и системы автоматизации энергосистем общего пользования. Часть 8-1. Схема распределения особой услуги связи (SCSM). Схема распределения для производственной системы модульной конструкции MMS (ISO 9506-1 и ISO 9506-2) и по ISO/IEC 8802-3

3. МЭК 61850-9-2 Системы автоматизации и сети связи на подстанциях. Часть 9-2. Схема особого коммуникационного сервиса (SCSM). Значения выборок по ISO/IEC 8802-3, - 2004, 34 с.

4. Международный стандарт организации ПАО «ФСК ЕЭС» № 96 // Типовые методики испытаний компонентов ЦПС на соответствие стандарту МЭК 61850 первой и второй редакций. М.: ПАО «ФСК ЕЭС», 2018. C. 87.

5. Осак А. Б. Человеческий фактор при обеспечении кибербезопасности объектов электроэнергетики / А. Б. Осак, Д. А. Панасецкий, Е. Я. Бузина, Исэм Со Ран // Современные направления развития систем релейной защиты и автоматики энергосистем: мат-лы V Межд. науч.-тех.конф. «Современные направления развития систем релейной защиты и автоматики энергосистем», Сочи, Россия, 1-5 июня 2015. Сочи, 2015. 6 с.

6. Khan R., Mclaughlin K., Laverty D., Sezer S. Design and Implementation of Security Gateway for Synchrophasor Based Real-Time Control and Monitoring in Smart Grid, Digital Object Identifier 10.1109/ ACCESS.2017.2716440

7. МЭК 61351 Управление энергетическими системами и связанный с этим обмен информацией. Безопасность данных и коммуникаций. Часть 7. Объектные модели данных сетей и систем управления - 2010. 44 c. URL: http://www.gostinfo.ru/catalog/Details/?id=4570716

8. Орлов Л. Л., Егоров Д. В. Опыт проектирования и внедрения систем РЗА и АСУТП на базе технологии МЭК 61850 // Промышленные АСУ и контроллеры. 2009. № 12. С. 41-44.

\section{REFERENCES AND INTERNET RESOURCES}

1. Anoshin A. O., Golovin A. V. Standart MJeK 61850. Protokol GOOSE // Novosti JelektroTehniki. 2012. № 6(78). Protokol MJeK 61850 Kommunikacionnye seti i sistemy podstancij.

2. MJeK 61850-8-1(2011) Seti svjazi i sistemy avtomatizacii jenergosistem obshhego pol'zovanija. Chast' 8,1 . Shema raspredelenija osoboj uslugi svjazi (SCSM). Shema raspredelenija dlja proizvodstvennoj sistemy modul'noj konstrukcii MMS (ISO 9506-1 i ISO 9506-2) i po ISO/IEC 8802-3.

3. MJeK 61850-9-2 Sistemy avtomatizacii i seti svjazi na podstancijah. Chast' 9,2, Shema osobogo kommunikacionnogo servisa (SCSM), Znachenija vyborok po ISO/IEC 8802-3. 2004, $34 \mathrm{~s}$.

4. Mezhdunarodnyj standart organizacii PAO «FSK EJeS» №96, Tipovye metodiki ispytanij komponentov CPS na sootvetstvie standartu MJeK 61850 pervoj i vtoroj redakcij. M.: PAO «FSK EEHS», 2018. S. 87.

5. Osak A. B. Chelovecheskij faktor pri obespechenii kiberbezopasnosti ob\#ektov jelektrojenergetiki (The human factor in ensuring the cybersecurity of power facilities) / A. B. Osak, D. A. Panaseckij, E. Ja. Buzina, Isjem So Ran // Sovremennye napravlenija razvitija sistem relejnoj zashhity i avtomatiki jenergosistem: mat-ly V Mezhd. nauch.-teh.konf. «Sovremennye napravlenija razvitija sistem relejnoj zashhity i avtomatiki jenergosistem», Sochi, Rossija, 1-5 ijunja 2015. Sochi, 2015. 6 s. 
6. Khan R., Mclaughlin K., Laverty D., Sezer S. Design and Implementation of Security Gateway for Synchrophasor Based Real-Time Control and Monitoring in Smart Grid, Digital Object Identifier 10.1109/ ACCESS.2017.2716440

7. MJeK 61351 Upravlenie jenergeticheskimi sistemami i svjazannyj s jetim obmen informaciej. Bezopasnost' dannyh i kommunikacij. Chast' 7. Ob\#ektnye modeli dannyh setej i sistem upravlenija, 2010. $44 \mathrm{~s}$.

8. Orlov L.L., Egorov D.V. Opyt proektirovanija i vnedrenija sistem RZA i ASUTP na baze tehnologii MJeK 61850 (Experience in the design and implementation of relay protection and automation systems and process control systems based on IEC 61850 technology) // Promyshlennye ASU i kontrollery. 2009. No 12. S. 41-44.

\section{СВЕДЕНИЯ ОБ АВТОРАХ}

Тучина Дарья Сергеевна, студент 2 курса магистратуры, кафедра автоматизированных электроэнергетических систем и электроснабжения, Северо-Кавказский федеральный университет. E-mail: tuchinads $(a)$ yandex.ru.

Старченко Алексей Валерьевич, студент 2 курса магистратуры, кафедра автоматизированных электроэнергетических систем и электроснабжения, Северо-Кавказский федеральный университет. E-mail: alexej.starchenko2017@yandex.ru.

Стребкова Татьяна Владимировна, студент 1 курса магистратуры, кафедра автоматизированных электроэнергетических систем и электроснабжения, Северо-Кавказский федеральный университет. E-mail: tanya.strebkova@mail.ru

Питько Юлия Михайловна, студент 1 курса магистратуры, группы 5АМ8Р, инженерная школа энергетики, Национально-исследовательский Томский политехнический университет. E-mail: julia14032007@mail.ru

\section{INFORMATION ABOUT AUTHORS}

Daria Tuchina, 2nd year student of the magistracy, Department of Automated Power Systems and Power Supply, Electrical Engineering and Electronics, North-Caucasian Federal University,E-mail: tuchinads $a$ yandex.ru.

Aleksey Starchenko, 2nd year student of the magistracy, Department of Automated Power Systems and Power Supply, Electrical Engineering and Electronics, North-Caucasian Federal University, E-mail: alexej. starchenko2017@yandex.ru.

Tatyana Strebkova, 1st year student of the magistracy, Department of Automated Power Systems and Power Supply, Electrical Engineering and Electronics, North-Caucasian Federal University. E-mail: tanya.strebkova@ @mail.ru

Yuliya Pitko, 1st year student of the magistracy, engineering school of energy, Tomsk Polytechnic University. E-mail: julia14032007@mail.ru 